Revista Electrónica Complutense de Investigación en Educación Musical ISSN-e: 1698-7454

http://dx.doi.org/10.5209/RECIEM.54828

\title{
Impacto de la educación musical: una revisión de la literatura científica'
}

\author{
Carmen Carrillo Aguilera²; Laia Viladot Vallverdú3 ${ }^{3}$ Jéssica Pérez-Moreno ${ }^{4}$.
}

Recibido: 25 de diciembre de 2016 / Aceptado: 14 de septiembre de 2017

Resumen. Este artículo estudia el impacto de la educación musical cursada en las enseñanzas obligatorias a partir de una revisión de la literatura académica existente. La búsqueda en bases de datos especializadas proporcionó un total de 25 artículos publicados durante el periodo 2005 - 2016. Tras analizarlos se establecieron dos categorías de impacto: DENTRO, en relación con la propia disciplina y FUERA, con implicaciones más allá de la disciplina. Buena parte de las publicaciones analizadas contemplaron el impacto de acuerdo con temáticas que sobrepasan lo que es estrictamente la educación musical obligatoria abarcando aspectos personales, intelectuales y/o sociales. En consecuencia, se presenta una conceptualización de impacto en educación musical que va más allá de los aspectos mensurables asociados a las políticas educativas de reforma.

Palabras clave: Impacto; educación musical; revisión de literatura; sociedad.

\section{[en] Impact of Music Education: a Review of the Scientific Literatura}

\begin{abstract}
This paper examines the impact of school music education through an academic literature review. The search in specialized databases provided a total of 25 papers published during $2005-2016$. The analysis revealed two categories in relation to impact: IN, that included those issues within the discipline, and OUT, with implications beyond it. Many of the analysed publications considered the impact based on topics that go beyond what strictly school music education is, including personal, intellectual and/or social aspects. This study presents a conceptualization of impact on music education that surpasses the measurable aspects associated with educational policy reforms.
\end{abstract}

Keywords: Impact; music education; literature review; society.

Sumario. 1. Introducción, 2. Metodología. 3. Resultados, 3.1. Visión general, 3.2. El impacto de la educación musical a través de las categorías establecidas. 3.2.1. Dentro de la disciplina, 3.2.2. Fuera de la disciplina, 3.2.2.1. Ámbito personal, 3.2.2.2. Ámbito intelectual, 4. Discusión, 5. Referencias.

1 Este artículo es parte del proyecto de investigación I+D EDU2014-58066-P sobre El impacto de la educación musical en la sociedad y en la economía del conocimiento, financiado por el Ministerio de Economía y Competitividad del Gobierno de España.

2 Universidad Internacional de Cataluña

ccarrillo@uic.es

3 Universidad Autónoma de Barcelona

laia.viladot@uab.cat

$4 \quad$ Universidad Autónoma de Barcelona

Jessica.Perez@uab.cat 
Cómo citar: Carrillo Aguilera, C.; Viladot Vallverdú, L.; Pérez-Moreno, J. (2017). Impacto en la educación musical: una revisión de la literatura científica, en Revista Electrónica Complutense de Investigación en Educación Musical, 14, 61-74.

\section{Introducción}

La educación musical es una materia que, en general, se contempla en los currículos de las enseñanzas obligatorias en la mayor parte de los países occidentales. Si bien aprender música es algo que tiene valor como un fin en sí mismo, de forma creciente su inclusión en el currículo escolar durante estos últimos años se ha venido justificando con base en sus beneficios para el aprendizaje de otras disciplinas más allá de la propia en cuestión.

En España, la enseñanza de la educación musical en las etapas educativas obligatorias se generalizó en la década de los 90. Sin embargo, el marco curricular vigente para la educación primaria desarrollado recientemente a partir de la Ley Orgánica para la Mejora de la Calidad Educativa (LOMCE; Jefatura del Estado, 2013), establece la educación artística como área opcional en el currículo de educación primaria y secundaria y, por tanto, introduce la posibilidad de que el alumnado acabe la educación obligatoria sin haber experimentado ningún tipo de práctica musical ni en relación con otras materias artísticas. En este contexto de reformas, surge IMPACTMUS, un proyecto I+D+i (EDU2014-58066-P) financiado por el Ministerio de Economía y Competitividad español que se propone indagar, a partir de datos empíricos, el impacto que la educación musical ha tenido en las enseñanzas obligatorias desde su generalización en las escuelas españolas en los 90 . Se ha escrito mucho sobre el impacto que el aprendizaje de la música ha tenido en las personas; sin embargo, muy pocos trabajos se han basado en evidencias empíricas $\mathrm{y}$, de estos, la mayor parte ha tenido en cuenta la música que se cursa más allá de la escuela (como materia extraescolar en conservatorios o escuelas de música) (Rickard, Bambrick y Gill, 2012).

Por ello, en el marco del proyecto IMPACTMUS, este artículo tiene por objeto realizar una revisión bibliográfica de la literatura que, a nivel internacional, analiza el impacto que la educación musical cursada en las enseñanzas obligatorias ha tenido durante esta última década en los estudiantes que la han llevado a cabo. En dicha revisión se han tenido en cuenta los trabajos de naturaleza empírica que desde 2005 han examinado cómo la educación musical cursada durante los niveles educativos obligatorios ha incidido en el propio alumnado y en la sociedad en general, tanto a nivel musical como más allá de la propia materia de música. Por tanto, en este artículo el término impacto se define como el conjunto de posibles efectos sobre las personas y la sociedad, como consecuencia de las actividades de índole musical llevadas a cabo en el aula de educación primaria y secundaria. Si bien el concepto de impacto en educación se encuentra fuertemente impregnado por el carácter economicista, cuantitativista, determinista y, en definitiva, neoliberal de las políticas educativas de reforma, este estudio presenta un enfoque basado en investigaciones que difieren en gran medida de esta conceptualización inicial. En él se pretende recopilar investigaciones sobre esta temática que permitan contribuir al debate acerca del papel que 
debe jugar la música en las enseñanzas obligatorias en el contexto actual de reformas en las políticas educativas.

\section{Metodología}

La revisión bibliográfica llevada a cabo focalizó en la literatura que durante el periodo 2005 - 2016 relacionó los términos music education e impact. La búsqueda se confinó a este periodo tras comprobar, en una primera revisión informal de literatura existente en diferentes bases de datos de educación, que la mayor parte de los trabajos publicados en torno a esta temática eran posteriores a 2005. Este hecho podría ser reflejo de la necesidad, por parte de la comunidad educativa durante esta última década, de aportar referentes sólidos que permitan plantearse el papel de la música en el currículo obligatorio en el marco de las políticas educativas de reforma.

En una primera fase del estudio se realizó una búsqueda en las bases de datos ERIC, SCOPUS y Web of Science. Se buscaron publicaciones que en su título, resumen o palabras clave (título o tema en el caso de Web of Science), contuvieran los términos music education e impact. En total se encontraron 221 publicaciones de las que, tras descartar las duplicadas y aquellas en idiomas desconocidos para las autoras (se analizaron artículos en inglés y español), sólo 11 cumplieron con los criterios de inclusión establecidos previamente para esta revisión bibliográfica. Dichos criterios guardan relación con la fuente de publicación (revistas académicas con establecidos procesos de revisión por pares), la naturaleza del trabajo (estudios de naturaleza empírica) y la relevancia de la temática (trabajos en torno al impacto de la educación musical cursada durante las enseñanzas obligatorias). Teniendo en cuenta los mismos criterios, en una fase posterior del estudio se procedió a una búsqueda manual a través de las referencias bibliográficas de los artículos previamente seleccionados. Este proceso generó un total de 14 artículos nuevos, no encontrados en las búsquedas anteriores.

Los trabajos seleccionados $(\mathrm{n}=25)$ se sometieron a dos tipos de análisis. Primeramente se analizaron de forma descriptiva. Para ello se construyó una tabla en la que se sintetizaron los aspectos que se consideraron más relevantes en relación con: el propósito de cada estudio, el contexto, las características de la muestra, la metodología y los principales resultados (véase un ejemplo en la Tabla 1). 
Tabla 1. Ejemplo del análisis descriptivo realizado

\begin{tabular}{|l|l|}
\hline Autor y año & Wong (2005) \\
\hline Propósito & $\begin{array}{l}\text { Explorar las opiniones de docentes de primaria acerca } \\
\text { de los elementos esenciales de la educación musical y su } \\
\text { impacto en los estudiantes }\end{array}$ \\
\hline Contexto. Muestra & $\begin{array}{l}\text { China y Canadá; } \mathrm{n}=10 \text { docentes de primaria en escuelas } \\
\text { públicas de Vancouver y Hong Kong (5 en cada contex- } \\
\text { to) }\end{array}$ \\
\hline Metodología & $\begin{array}{l}\text { 10 estudios de caso; observaciones no participantes y en- } \\
\text { trevistas }\end{array}$ \\
\hline Principales resultados & $\begin{array}{l}\text { Los docentes de ambos contextos mantienen opiniones } \\
\text { parecidas acerca de los elementos esenciales de la edu- } \\
\text { cación musical, pero difieren en cuanto a su impacto en } \\
\text { los estudiantes. } \\
\text { Mientras que la mayor parte de ellos coinciden en que } \\
\text { el propósito de la educación musical es proporcionar a } \\
\text { los estudiantes experiencias musicales que les permitan } \\
\text { desarrollar las capacidades para apreciar y hacer músi- } \\
\text { ca, los docentes chinos creen además que dicha materia } \\
\text { debe proporcionarles conocimientos musicales con los } \\
\text { que enriquecer su temperamento y carácter. }\end{array}$ \\
\hline
\end{tabular}

En una fase posterior del estudio se analizaron los resultados de cada uno de los artículos seleccionados con el propósito de establecer patrones y aportar datos que adquirieran significado más allá de cada caso específico (Huberman y Miles, 1994), sin obviar las características particulares de cada uno de ellos. Concretamente, el procedimiento de análisis para construir conocimiento de forma emergente (inspirándose en la Teoría Fundamentada de Glaser y Strauss, 1967) se desarrolló de la siguiente manera: las tres autoras analizaron los 25 artículos de la tabla por separado y propusieron unas categorías. Como hubo discrepancias entre ellas en las propuestas de clasificación, el diálogo (explicitando criterios, motivos, interpretaciones, entre otros aspectos) y la reflexión conjunta fueron necesarios hasta llegar al consenso. Fruto de este proceso de análisis y de construcción emergente, se establecieron dos grandes categorías que hacen referencia al impacto de la educación musical en el seno de la propia disciplina (impacto $D E N T R O$ ) y más allá de la disciplina (impacto FUERA). A su vez, los resultados en relación con el impacto FUERA se organizaron en tres subcategorías que corresponden al impacto de la educación musical en el ámbito personal, social e intelectual de cada individuo (v. Tabla 2 para un listado de los artículos analizados con las correspondientes categorizaciones). 
Tabla 2. Correspondencia de los artículos con las categorías y subcategorías

\begin{tabular}{|c|c|c|c|c|}
\hline \multirow[b]{2}{*}{ Artículos analizados } & \multirow[b]{2}{*}{ DENTRO } & \multicolumn{3}{|c|}{ FUERA } \\
\hline & & 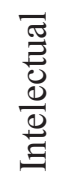 & 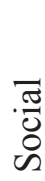 & 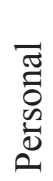 \\
\hline Abril y Gault (2008) & $\checkmark$ & & $\checkmark$ & $\checkmark$ \\
\hline Andreu y Godall (2012) & & $\checkmark$ & & \\
\hline Cabedo-Mas y Díaz-Gómez (2013) & & & $\checkmark$ & \\
\hline Campbell, Connell y Beegle (2007) & $\checkmark$ & & $\checkmark$ & $\checkmark$ \\
\hline Crawford (2016) & & & $\checkmark$ & $\checkmark$ \\
\hline Eerola y Eerola (2014) & & & & $\checkmark$ \\
\hline Gillespie, Russell y Hamann (2014) & $\checkmark$ & & $\checkmark$ & \\
\hline Hallam, Creech y McQueen (2015) & $\checkmark$ & & $\checkmark$ & \\
\hline $\begin{array}{l}\text { Hernández-Bravo, Cardona-Moltó y Hernán- } \\
\text { dez-Bravo (2016) }\end{array}$ & $\checkmark$ & & & \\
\hline Hinshaw et al. (2015) & & & $\checkmark$ & \\
\hline Hogenes et al. (2016) & $\checkmark$ & $\checkmark$ & & \\
\hline Johnson y Memmott (2006) & & $\checkmark$ & & \\
\hline Kokotsaki y Hallam (2007) & $\checkmark$ & & $\checkmark$ & $\checkmark$ \\
\hline Kokotsaki y Hallam (2011) & $\checkmark$ & & $\checkmark$ & $\checkmark$ \\
\hline Marsch (2012) & & & & $\checkmark$ \\
\hline Miksza (2010) & & $\checkmark$ & & $\checkmark$ \\
\hline Osborne et al. (2016) & & $\checkmark$ & $\checkmark$ & $\checkmark$ \\
\hline Pitts (2009) & & & $\checkmark$ & \\
\hline Portowitz, Peppler y Downton (2014) & $\checkmark$ & $\checkmark$ & & \\
\hline Rickard, Bambrick y Gill (2012) & & $\checkmark$ & $\checkmark$ & $\checkmark$ \\
\hline Rickard et al. (2010) & & $\checkmark$ & & \\
\hline Rickard et al. (2013) & & & $\checkmark$ & $\checkmark$ \\
\hline Seifried (2006) & $\checkmark$ & & $\checkmark$ & \\
\hline Valenzuela y Codina (2014) & $\checkmark$ & & & \\
\hline Wong (2005) & $\checkmark$ & & $\checkmark$ & \\
\hline
\end{tabular}

A continuación se presentan los resultados de ambos análisis: el primero proporciona una visión general de la literatura seleccionada en torno al impacto de la educación musical en las enseñanzas obligatorias y el segundo presenta los resultados 
de cada artículo en relación con cada una de las categorías establecidas referentes al impacto.

\section{Resultados}

\subsection{Visión general}

En este punto se discuten los resultados más relevantes en relación con el propósito, el contexto, las características de la muestra y la metodología de los artículos seleccionados.

En primer lugar se pudo comprobar que, aunque el propósito de nuestro estudio es analizar la literatura existente que examina el impacto que la educación musical que se cursa en las enseñanzas obligatorias tiene sobre aquellos individuos que la llevan a cabo, casi la mitad de los artículos analizados contemplaron aspectos que van más allá de la materia estándar que se ofrece en estos niveles educativos. Es decir, siete examinaron sus efectos tras la implementación de un programa o proyecto específico (Gillespie, Russell y Hamann, 2014; Hallam, Creech y McQueen, 2015; Hernández-Bravo, Cardona-Moltó y Hernández-Bravo, 2016; Hinshaw et al. 2015; Seifried, 2006; ; Osborne et al., 2016; Portowitz, Peppler y Downton, 2014) y cinco analizaron el impacto de un programa musical ampliado o intensificado (Andreu y Godall, 2012; Eerola y Eerola, 2014; Rickard, Appelman et al., 2013; Rickard, Bambrick y Gill, 2012; Rickard, Vasquez et al., 2010). Del resto de artículos, que sí contemplaron la educación musical que se cursa de forma habitual en las enseñanzas obligatorias, tres se centraron en la influencia que las experiencias musicales recibidas a lo largo de la vida (lo que incluye también aquellas durante el periodo escolar) ejercieron sobre su identidad personal o profesional futura (Kokotsaki y Hallam, 2007, 2011; Pitts, 2009) y dos analizaron el impacto que tuvieron actividades concretas de la materia y no toda la materia en su conjunto (Hogenes et al., 2016; Miksza, 2010).

En segundo lugar se constató que la mayor parte de los estudios se habían llevado a cabo en contextos anglosajones, tanto en Estados Unidos de Norteamérica $(n=6)$ y en Australia $(\mathrm{n}=6)$, como en Reino Unido $(\mathrm{n}=5)$, países en los que quizás las políticas educativas de reforma han tenido una mayor incidencia sobre la educación musical cursada en las enseñanzas obligatorias. De estas investigaciones, merecen especial mención las lideradas o desarrolladas por Hallam y Rickard, y sus respectivos colaboradores (Hallam, Creech y McQueen, 2015; Kokotsaki y Hallam, 2007; 2011; Rickard, Appelman et al., 2013; Rickard, Bambrick y Gill, 2012; Rickard, Vasquez et al., 2010). Sin embargo, es destacable que cuatro de las investigaciones analizadas se desarrollaron completa o parcialmente en España, lo que quizás es una señal de la inquietud existente, por parte de algunos miembros de la comunidad educativa española, en relación con el papel que debe jugar dicha materia en el currículo escolar obligatorio en el contexto actual de reformas.

En tercer lugar, en relación con la naturaleza de la muestra empleada, se pudo detectar que 14 estudios focalizaron en las percepciones o resultados en relación con distintos aspectos de muestras, en general, amplias de alumnos de educación 
primaria o secundaria (oscilan entre 14 y 12.160, siendo más de la mitad superiores a 200 estudiantes), ocho tuvieron en cuenta las opiniones o experiencias de otros miembros implicados directa o indirectamente en la formación musical de dichos alumnos y tres contemplaron percepciones y/o experiencias tanto de un colectivo como del otro.

Finalmente, en lo que concierne a cuestiones metodológicas, es destacable que poco más de la mitad de los artículos examinados $(n=13)$ utilizó exclusivamente instrumentos como pruebas, cuestionarios o escalas de medición diversas para recopilar datos, mayoritariamente de carácter cuantitativo, que permitieran evaluar el impacto de la educación musical obligatoria en relación con aspectos del alumnado de distinta índole. Por otro lado, ocho estudios emplearon técnicas de recogida de datos como entrevistas, observaciones o cuestionarios de respuesta abierta, entre otras, para obtener datos de naturaleza cualitativa que permitieran valorar la influencia de esta materia en aquellos estudiantes que la habían cursado. Los cuatro artículos restantes combinaron técnicas que facilitaron el compendio de datos tanto de carácter cuantitativo como cualitativo.

\subsection{El impacto de la educación musical a través de las categorías establecidas}

\subsubsection{Dentro de la disciplina}

De los artículos analizados, 12 focalizaron en el impacto que la educación musical obligatoria ejerce en la propia disciplina (ver categoría DENTRO en Tabla 2); sin embargo, sólo dos de ellos lo hicieron de manera exclusiva. Hernández-Bravo, Cardona-Moltó y Hernández-Bravo (2016) estudiaron la influencia que tuvo, sobre diferentes aspectos musicales, una intervención en el aula a partir de programas informáticos. Los resultados pusieron de manifiesto que aquellos alumnos con un dominio musical medio y alto fueron quienes se beneficiaron más de la implementación de este programa y quienes consiguieron desarrollar en mayor medida los distintos aspectos musicales investigados (en relación con la audición, la expresión vocal e instrumental, el lenguaje musical, el movimiento y la danza, entre otros). Por su parte, Valenzuela y Codina (2014) evidenciaron que las experiencias musicales que reportó el alumnado que proviene de familias con bagaje musical fueron mucho más positivas que aquellos cuyas familias carecen de dicho bagaje.

Del resto de artículos, un número importante de ellos enfatizaron la mejora que supone la educación musical recibida en la escuela para una serie de aspectos relacionados con las competencias perceptivas y expresivas del alumnado. Al explorar las percepciones de una muestra de docentes de diferentes contextos culturales en torno al impacto de la educación musical obligatoria sobre los estudiantes, Wong (2005), por ejemplo, puso de manifiesto su importancia para el desarrollo de capacidades para apreciar y hacer música. Desde ópticas distintas, también Abril y Gault (2008), Campbell, Connell y Beegle (2007), Gillespie, Russell y Hamann (2014), Hallam, Creech y McQueen (2015), Kokotsaki y Hallam (2007, 2011) y Seifried (2006) se centraron en el impacto que la educación musical tuvo para el desarrollo de competencias relacionadas con la interpretación y/o la escucha musical. De forma complementaria, Hallam, Creech y McQueen (2015) y Portowitz, Peppler y Dowton 
(2014) pusieron énfasis en cómo las respectivas intervenciones consiguieron mejorar la comprensión musical del alumnado.

Abril y Gault (2008) proporcionaron una perspectiva diferente sobre el impacto de los programas de educación musical en las competencias musicales del alumnado. Al preguntar a los directores de una muestra amplia de escuelas estadounidenses acerca de su percepción en torno a dicho impacto, éstos consideraron las competencias en relación con la escucha y la interpretación musical como las que la educación musical escolar consiguió desarrollar en mayor medida. En el polo opuesto, la competencia relacionada con la creación fue la que, en su opinión, los alumnos desarrollaron menos. También sobre estas competencias, aunque desde una óptica distinta, Hogenes et al. (2016) revelaron que la educación musical más centrada en la producción musical conduce a una mayor implicación musical por parte del alumnado que aquella que lo hace en la reproducción.

Finalmente, en los artículos analizados también se evidenció la importancia de la educación musical como medio para comprender la sociedad y la cultura, tanto de aquellas en las que uno se encuentra inmerso (Campbell, Connell y Beegle, 2007), como las ajenas (Gillespie, Russell y Hamann, 2014).

\subsubsection{Fuera de la disciplina}

De los artículos analizados, la gran mayoría (23) trata el impacto de la educación musical fuera de la disciplina, y lo hacen en relación con tres ámbitos: el personal, el intelectual y el social (ver categoría FUERA y sus subcategorías en Tabla 2).

\subsubsection{1. Ámbito personal}

El impacto que la educación musical obligatoria tiene sobre la dimensión personal del individuo se reveló como una de las más importantes en las investigaciones analizadas. En total, 15 artículos trataron esta temática, ya sea como objeto principal o secundario. De estos, un número elevado se centró en aspectos en torno al bienestar psicológico del alumnado. Así, por ejemplo, los datos aportados por Hinshaw et al. (2015) al analizar la influencia de un grupo de canto en los estudiantes británicos, evidenciaron un abanico de beneficios en su bienestar; por ejemplo, sentimientos de gozo o de entusiasmo. Kokotsaki y Hallam (2007; 2011), que indagaron el impacto que había tenido la participación activa en actividades musicales a lo largo de la vida para una muestra de estudiantes universitarios, hallaron que su implicación en grupos de interpretación musical en el pasado (también durante la etapa escolar) mejoró sus niveles de autoestima y autoconfianza, revirtiendo en una mayor satisfacción personal. Además de estos autores, los estudios llevados a cabo por Abril y Gault (2008), Crawford (2016), Gillespie, Russell y Hamman (2014), Hallam, Creech y McQueen (2015), Osborne et al. (2016) y Rickard y colaboradores (Rickard, Bambrick y Gill, 2012; Rickard, Appelman et al., 2013) también pusieron de manifiesto un impacto positivo en aspectos relacionados con la autoestima y/o la autoconfianza del alumnado que había cursado educación musical en las enseñanzas obligatorias. Sin embargo, tanto Hinshaw et al. (2015) como Rickard, Bambrick y Gill (2012) hallaron que el impacto sobre la autoestima del alumnado fue sutil, especialmente para aquellos estudiantes con un nivel de bienestar elevado. 
El impacto personal a nivel identitario fue también relevante. Dicho impacto se contempló desde dos perspectivas distintas: por un lado, Campbell, Connell y Beegle (2007), Kokotsaki y Hallam (2007) y Seifried (2006) se refirieron a la música cursada en la escuela (y fuera de la escuela en el primer estudio) como parte integral de su identidad personal y/o colectiva y que, además, ayudó a forjar su personalidad. Una visión complementaria se puso de manifiesto en el estudio de Wong (2005), en el que una parte importante de los docentes participantes consideró que la educación musical debía proporcionar al alumnado conocimientos musicales con los que enriquecer su temperamento y carácter. Por otro lado, las investigaciones de Cabedo-Mas y Díaz-Gómez (2013) y Pitts (2009) enfatizaron cómo las influencias musicales positivas en las aulas (y fuera de ellas en el caso de Pitts) contribuyeron a la implicación activa de los individuos en actividades de carácter musical a lo largo de sus vidas. En Pitts, además, se señaló al docente de música como la persona más inspiradora y la que influyó en mayor medida en este sentido.

Otras investigaciones incidieron en la influencia que la educación musical tiene a nivel emocional. Por ejemplo, Campbell, Connell y Beegle (2007), Gillespie, Russell y Hamann (2014) y Seifried (2006), describieron cómo la música fue para los individuos implicados en ella un vehículo para el gozo personal y/o un instrumento terapéutico para relajarse o liberar tensiones. Los dos primeros estudios, además, aludieron a los beneficios de la educación musical como herramienta para desarrollar aptitudes personales como la autodisciplina. Se considera relevante destacar, no obstante, que dichos estudios contemplaron también la música cursada más allá de las aulas escolares (Campbell, Connell y Beegle, 2007) o la implementación de programas musicales que sobrepasan lo que podría considerarse común en el marco de la educación musical en las enseñanzas obligatorias (Gillespie, Russell y Hamann, 2014; Seifried, 2006). Finalmente, otros dos estudios en los que se introdujeron proyectos musicales específicos también reportaron resultados muy positivos relacionados con la motivación del alumnado implicado (Hallam, Creech y McQueen, 2015; Hinshaw, Clift, Hulbert y Camic, 2015).

\subsubsection{2. Ámbito intelectual}

En el ámbito intelectual se recogen aquellos trabajos que aportan evidencias sobre el impacto de la educación musical en aspectos cognitivos y académicos. Se encuentran 8 trabajos que tienen por objetivo estudiar estas dimensiones del individuo, y lo hacen a través de pruebas o test estandarizados. Por un lado están Andreu y Godall (2012) que aportan evidencias sobre cómo la educación musical ayuda a la adquisición de competencias básicas (sobre todo en el ámbito matemático, socionatural y en el trabajo cooperativo). En esta misma línea pero en contexto norteamericano, la investigación de Johnson y Memmott (2006) ya había afirmado unos años antes que existía relación entre una enseñanza de la música de calidad y los logros académicos, sobre todo en relación con las matemáticas. Los estudios de Miksza (2010), Osborne et al. (2016) y Rickard, Bambrick y Gill (2012) reiteran esta idea de la relación con las matemáticas para un amplio abanico de población (etapas de secundaria y primaria respectivamente).

Por otro lado, concerniente a aspectos cognitivos, encontramos a Rickard, Vasquez, Murphy et al. (2010) quienes afirman que el incremento de clases de formación 
instrumental tiene efectos en funciones cognitivas no-musicales, como la memoria verbal (apoyada también por Osborne et al., 2016) y la memoria de trabajo a corto plazo (apoyada también por Portowitz, Peppler y Downton, 2014). Estos últimos autores aportan datos, además, para mostrar que la educación musical tiene efectos en la autorregulación del aprendizaje y la flexibilidad cognitiva del alumnado. Finalmente, y como contrapunto a los artículos presentados en esta sección, se encuentra una discrepancia en torno a los posibles efectos en la inteligencia no-verbal; Osborne et al. (2016) afirman que la educación musical contribuye a esta capacidad, sin embargo Hogenes et al. (2016) no apoyan esta hipótesis.

\subsubsection{3. Ámbito social}

Esta subcategoría reúne 11 de los artículos analizados, aunque solamente dos trabajos se proponen analizar -de forma exclusiva- los beneficios sociales de la educación musical, estos son Eerola y Eerola (2013) y Marsh (2015). A pesar de estudiar dos contextos muy distintos; los primeros en Finlandia con alumnos de diferentes centros de primaria con más o menos educación musical, y el otro centrado en refugiados e inmigrantes jóvenes en Sydney, a ambos les une la constatación de que la educación musical mejora la convivencia y la satisfacción dentro del centro escolar. Y de forma muy específica, Marsh añade el matiz de sentirse integrado y formar parte de un colectivo. Esta idea de conocer gente y formar parte de un colectivo la habían relatado también estudiantes universitarios del Reino Unido en Kokotsaki y Hallam (2011).

Otros artículos que también hacen hincapié en la dimensión social, aunque no de forma exclusiva, hablan de los beneficios que aporta la educación musical para el trabajo cooperativo y en equipo (Abril y Gault, 2008; Kokotsaki y Hallam, 2007) y de cómo este trabajo en equipo repercute en la autoestima (Abril y Gault, 2008) y en el sentirse apreciado por los demás (Kokotsaki y Hallam, 2011).

De forma complementaria también existen los trabajos que abren una mirada más amplia hacia el concepto de responsabilidad social (Osborne et al. 2016), en el sentido de que están más concienciados con los asuntos de carácter ético de la comunidad, como construir amistades, ayudar a los otros, corregir desigualdades sociales, la inclusión social en relación al sentimiento de pertenencia al colectivo, entre otros (Miksza, 2010; Crawford, 2016), así como el rol que ejerce la educación musical (y la música) en adolescentes estadounidenses como "cumplimiento de sus necesidades sociales", en el sentido de que les distrae de conductas sociales no apropiadas (Campbell et al., 2007).

Finalmente, en contraposición a estos trabajos expuestos relacionados con el ámbito social, Rickard, Appelman, James et al. (2013) explican que incrementar horas de formación musical no demostró tener beneficios sobre las habilidades sociales, y Rickard, Bambrick y Gill (2013) matizan que las evidencias sobre beneficios de la educación musical cursada en la enseñanza obligatoria a nivel psicosocial son muy sutiles en su estudio con alumnado australiano de 10 a 13 años. Parece ser que cuanto más desfavorecido es el contexto, más impacto tiene en la población la educación musical. 


\section{Discusión}

Se articulan a modo de discusión final las aportaciones de este trabajo bajo dos perspectivas: por un lado, se pone en valor la categorización per se presentada y, por otro, se exponen aquellas ideas y reflexiones fruto del análisis del contenido de los artículos estudiados.

Esta revisión de la literatura pone de relieve la escasez de trabajos que aportan evidencias empíricas sobre el impacto de la educación musical en las enseñanzas obligatorias. Se ha visto que casi la mitad de los artículos analizados contemplan aspectos de ampliación de la materia estándar que se ofrece en estos niveles educativos. Es decir, se encuentran artículos que examinan los efectos tras la implementación de un programa o proyecto específico y otros que analizan el impacto de un programa musical ampliado o intensificado.

Otra aportación del presente trabajo es la propia categorización de dicha producción científica y la explicitación del proceso seguido para llegar a ésta. Como se expone en la metodología, fue a través del análisis cualitativo de contenido y de un laborioso proceso de validación interna que se llega a formular una de las primeras propuestas de categorización del impacto en relación con la educación musical escolar. Si bien las tres subcategorías coinciden en buena medida con las que utiliza Hallam (2010) en su trabajo sobre el impacto de la música en los niños y jóvenes, que es un referente en este campo, en general, las publicaciones de Hallam y colaboradores tienen en cuenta el impacto de la música en un sentido amplio y no sólo aquel que es fruto de la educación musical que se imparte en las enseñanzas obligatorias, como es el caso del trabajo que se presenta.

Respecto al contenido analizado de los artículos, se puede afirmar que -puesto que existen tan pocos estudios que examinen directamente los resultados dentro de la misma disciplina- la justificación de la inclusión de la educación musical en el currículo escolar pasa, muy a menudo, por indagar los beneficios (o efectos) de ésta en el aprendizaje de otras materias. En cambio, un número importante de trabajos trata aspectos relacionados con el impacto de la educación musical en la dimensión personal del individuo, con especial relevancia aquellos que examinan el bienestar psicológico del alumnado. Esto quizás es una muestra de que más allá de su valor intrínseco, la música en el currículo escolar deba justificarse en base a su valor personal.

De hecho, lo intrínseco y lo personal (que conciernen a la categoría DENTRO y a la subcategoría Personal / aspectos de identidad, respectivamente) van de la mano; los límites son difusos. Se ha visto que lo personal, en estos casos, sería consecuencia de un impacto previo en relación a DENTRO de la disciplina. Es decir, uno forja su identidad con base en experiencias musicales que han sido significativas y que por tanto han tenido un impacto previo a nivel musical. Son categorías que se retroalimentan; además, quizás un impacto positivo a nivel identitario puede revertir en mayores beneficios a nivel musical (por ejemplo, se puede pensar que si uno tiene experiencias muy positivas que hagan que la música sea algo intrínseco en él, quizás pondrá más atención y estará más motivado en lo que hace en la escuela en relación con la música y esto le lleve a aprender más y mejor).

También, dentro de la disciplina, se ha visto que las investigaciones ponen énfasis en cómo la educación musical ayuda a desarrollar las competencias interpretativas y perceptivas, pero no se alude casi a la creación. Quizás esto sea reflejo del modelo 
reproductivo todavía imperante en la educación musical (sobre todo fuera del ámbito anglosajón), a pesar de que las tendencias parecen estar cambiando. Uno de los estudios concluye que para que la educación musical tenga un mayor impacto debe estar basada más en la producción que en la reproducción (Hogenes et al., 2016). Quizás sea ésta también una de las claves para que la educación musical tenga un impacto sobre la sociedad; más producción y menos reproducción.

Por otro lado, ahora fuera de la disciplina, se ha hecho evidente con esta revisión que ninguna de las investigaciones realizadas en contexto español se focaliza en la dimensión social, esto es, en indagar el impacto que la educación musical escolar pueda tener en la sociedad. En este sentido, se valora la pertinencia del proyecto IMPACTMUS que se está llevando a cabo con este propósito. De forma muy específica, y como consecuencia de lo expuesto en esta sección, se anima a realizar estudios que indaguen en la dimensión personal desde el punto de vista de la identidad desde una visión retrospectiva de los ciudadanos para ver qué actividades contribuyen en mayor medida a una implicación activa en actividades de carácter musical a lo largo de la vida. Esta aproximación podría dar pistas sobre la orientación que debería seguir la educación musical cursada en la enseñanza obligatoria para tener un impacto real sobre la sociedad.

En el periodo de políticas de reforma educativa en el que estamos inmersos, se requieren argumentos y reflexiones que contribuyan al debate acerca del papel que debe jugar la música en las enseñanzas obligatorias. En este trabajo se ha puesto de manifiesto que el impacto en educación musical no puede regirse solamente por los criterios de efectividad que se han asociado a dichas políticas de reforma, fruto de la influencia que el neoliberalismo ha tenido en educación. El impacto en educación musical no puede - ni debe - ser exclusivamente mensurable pues dicho enfoque es difícilmente aplicable a una materia cuya esencia es de naturaleza artística y estética (Aróstegui, Louro y Teixeira, 2015). El concepto de impacto que se ha presentado tiene un fuerte componente personal, ligado al bienestar y a la satisfacción del alumnado, aunque también un valor intrínseco relacionado con el potencial de la música en el desarrollo de la creatividad y la innovación, esenciales para hacer frente a los retos que plantea la economía del conocimiento.

\section{Referencias}

Abril, C.R. \& Gault, B.M. (2008). The State of Music in Secondary Schools. The Principal's Perspective. Journal of Research in Music Education, 56(1), 68-81.

Andreu, M. \& Godall, C. (2012). La importancia de la educación artística en la enseñanza obligatoria: la adquisición de las competencias básicas de Primaria en un centro integrado de música. Revista de Educación, 357, 179 - 202.

Aróstegui, J.L.; Louro, A.L. \& Teixeira, Z.L. (2015). Las políticas educativas de reforma y su impacto en la Educación Musical Escolar. De dónde venimos y hacia dónde podemos ir. Revista da ABEM, 23(35), 24-34.

Cabedo-Mas, A. \& Díaz-Gómez, M. (2013) Positive musical experiences in education: music as a social praxis. Music Education Research, 15(4), 455-470. DOI: 10.1080/14613808.2013.763780. 
Campbell, P.; Connell, C. \& Beegle, A. (2007). Adolescents' expressed meanings of music in and out of school. Journal of Research in Music Education, 55(3), 220-36.

Crawford, R. (2016). Creating unity through celebrating diversity: A case study that explores the impact of music education on refugee background students. International Journal of Music Education, 1-14. DOI: 10.1177/0255761416659511.

Eerola, P.S. \& Eerola, T. (2014). Extended music education enhances the quality of school life. Music Education Research, 16(1), 88-104.

Gillespie, R.; Russell, J.A. \& Hamann, D.L. (2014) String Music Educators’ Perceptions of the Impact of New String Programs on Student Outcomes, School Music Programs, and Communities. Journal of Research in Music Education, 62(2), 175- 187.

Glaser, B.G. \& Strauss, A.L. (1967) The discovery of grounded theory: strategies for qualitative research. Chicago: Aldine.

Hallam, S. (2010). The power of music: Its impact on the intellectual, social and personal development of children and young people. International Journal of Music Education, 28(3) 269-289.

Hallam, S.; Creech, A. \& McQueen, H. (2015): Teachers' perceptions of the impact on students of the Musical Futures approach. Music Education Research, 1- 13. DOI: 10.1080/14613808.2015.1108299.

Hernández-Bravo, J.R.; Cardona-Moltó, M.C. \& Hernández-Bravo, J.A. (2016). The effects of an individualised ICT-based music education programme on primary school students' musical competence and grades. Music Education Research, 18(2), 176-194. DOI: 10.1080/14613808.2015.1049255.

Hinshaw, T.; Clift, S.; Hulbert, S. \& Camic, P.M. (2015). Group singing and young people's psychological well-being. International Journal of Mental Health Promotion, 17(1), 4663. DOI: 10.1080/14623730.2014.999454.

Hogenes, M.; van Oers, B.; Diekstra, R.F.W \& Sklad, M. (2016). The effects of music composition as a classroom activity on engagement in music education and academic and music achievement: A quasi-experimental study. International Journal of Music Education, $34(1), 32-48$.

Jefatura del Estado (2013). Ley Orgánica 8/2013 de 9 de diciembre, para la mejora de la calidad educativa (LOMCE). Boletín Oficial del Estado (10 de diciembre).

Johnson, C. \& Memmott, J. (2006). Examination of relationships between participation in school music programs of differing quality and standardized test results. Journal of Research in Music Education, 54(4), 293-307.

Kokotsaki, D. \& Hallam, S. (2007). Higher Education music students' perceptions of the benefits of participative music making. Music Education Research, 9(1), 93-109.

Kokotsaki, D. \& Hallam, S. (2011). The perceived benefits of participative music making for non-music university students: a comparison with music students. Music Education Research, 13(2), 149-172.

Marsh, C. (2012). The beat will make you be courage: The role of a secondary school music program in supporting young refugees and newly arrived immigrants in Australia. Research Studies in Music Education, 34(2), 93-111.

Miksza, P. (2010). Investigating relationships between participation in high school music ensembles and extra-musical outcomes: An analysis of the Education Longitudinal Study of 2002 using bio-ecological development model. Bulletin of the Council for Research in Music Education, 186, 7-25.

Osborne, M.S.; McPherson, G.E.; Faulkner, R.; Davidson, J. W. \& Barrett, M.S. (2016) Exploring the academic and psychosocial impact of El Sistema-inspired music programs wi- 
thin two low socio-economic schools. Music Education Research, 18(2), 156-175. DOI: 10.1080/14613808.2015.1056130.

Pitts, S. (2009). Roots and Routes in Adult Musical Participation: Investigating the Impact of Home and School on Lifelong Musical Interest and Involvement. British Journal of Music Education, 26(3), 241-256. doi:10.1017/S0265051709990088.

Portowitz, A.; Peppler, K.A. \& Downton, M. (2014). In Harmony: A Technology-Based Music Education Model to Enhance Musical Understanding and General Learning Skills. International Journal of Music Education, 32(2) 242-260.

Rickard, N.S.; Appelman, P.; James, R.; Murphy, F.; Gill, A. \& Bambrick, C. (2013). Orchestrating life skills: The effect of increased school-based music classes on children's social competence and self-esteem. International Journal of Music Education, 31(3), 292-309.

Rickard, N.S.; Bambrick, C.J. \& Gill, A. (2012). Absence of Widespread Psychosocial and Cognitive Effects of School-Based Music Instruction in 10-13-Year-Old Students. International Journal of Music Education, 30(1), 57-78.

Rickard, N.S.; Vasquez, J.T.; Murphy, F.; Gill, A. \& Toukhsati, S.R. (2010). Benefits of a classroom based music program on verbal memory of primary school children: a longitudinal study. Australian Journal of Music Education, 1, 36-47.

Seifried, S. (2006). Exploring the outcomes of rock and popular music instruction in high school guitar class: a case study. International Journal of Music Education, 24(2), 168 $-177$.

Valenzuela, R. \& Codina, N. (2014) Habitus and flow in primary school musical practice: relations between family musical cultural capital, optimal experience and music participation. Music Education Research, 16(4), 505-520. DOI: 10.1080/14613808.2013.859660.

Wong, M. (2005). A cross-cultural comparison of teachers' expressed beliefs about music education and their observed practices in classroom music teaching. Teachers and Teaching: theory and practice, 11(4), 397-418. 